\title{
Prevalence and antibiotic susceptibility pattern of CTX-M type extended-spectrum $\beta$-lactamases among clinical isolates of gram-negative bacilli in Jimma, Ethiopia
}

Ahmed Zeynudin ${ }^{1,2,3 \dagger}$, Michael Pritsch ${ }^{1,4,5+}$ O , Sören Schubert ${ }^{1}$, Maxim Messerer ${ }^{6}$, Gabriele Liegl ${ }^{1}$, Michael Hoelscher ${ }^{3,4,5}$, Tefara Belachew ${ }^{2}$ and Andreas Wieser ${ }^{1,2,4,5^{*}}$

\begin{abstract}
Background: The prevalence of extended-spectrum $\beta$-lactamases (ESBLs) have been reported in clinical isolates obtained from various hospitals in Ethiopia. However, there is no data on the prevalence and antibiotic susceptibility patterns of CTX-M type ESBL produced by Gram-negative bacilli. The aim of this study was to determine the frequency and distribution of the bla $a_{\mathrm{CTX}-\mathrm{M}}$ genes and the susceptibility patterns in ESBL producing clinical isolates of Gram-negative bacilli in Jimma University Specialized Hospital (JUSH), southwest Ethiopia.

Methods: A total of 224 non-duplicate and pure isolates obtained from clinically apparent infections, were included in the study. Identification of the isolates was performed by MALDI-TOF mass spectrometry. Susceptibility testing and ESBL detection was performed using VITEK ${ }^{\circledR}$ 2, according to EUCAST V4.0 guidelines. Genotypic analysis was performed using Check-MDR CT103 Microarrays.

Results: Of the total 112 (50.0\%) isolates screen positive for ESBLs, 63.4\% (71/112) tested positive for ESBL encoding genes by Check-MDR array, which corresponds to $91.8 \%$ (67/73) of the total Enterobacteriaceae and 10.3\% (4/39) of nonfermenting Gram-negative bacilli. Among the total ESBL gene positive isolates, 95.8\% (68/71) carried bla CTX-M genes with CTX-M group 1 type15 being predominant (66/68; $97.1 \%$ of CTX-M genes). The bla $a_{C T X-M}$ carrying Enterobacteriaceae $(n=64)$ isolates showed no resistance against imipenem and meropenem and a moderate resistance rate against tigecycline (14.1\%), fosfomycin (10.9\%) and amikacin (1.6\%) suggesting the effectiveness of these antibiotics against most isolates. On the other hand, all the bla $a_{\text {стX-м }}$ positive Enterobacteriaceae showed a multidrug resistant (MDR) phenotype with remarkable co-resistances (non-susceptibility rates) to aminoglycosides (92.2\%), fluoroquinolones (78.1\%) and trimethoprim/sulfamethoxazol (92.2\%).

Conclusions: This study demonstrates a remarkably high prevalence of bla CTX-M genes among ESBL-producing isolates. The high level of resistance to $\beta$-lactam and non- $\beta$-lactam antibiotics as well as the trend to a MDR profile associated with the bla $a_{\mathrm{CTX}-\mathrm{m}}$ genes are alarming and emphasize the need for routine diagnostic antimicrobial susceptibility testing for appropriate choice of antimicrobial therapy.
\end{abstract}

Keywords: Gram-negative bacilli, Extended-spectrum beta-lactamase, CTX-M, Antimicrobial susceptibility, Ethiopia

\footnotetext{
* Correspondence: wieser@mvp.uni-muenchen.de

${ }^{+}$Ahmed Zeynudin and Michael Pritsch contributed equally to this work

${ }^{1}$ Chair of Medical Microbiology and Hospital Epidemiology, Max von

Pettenkofer Institute, Faculty of Medicine, LMU Munich, Marchioninistr. 17,

81377 Munich, Germany

${ }^{2}$ Institute of Health Sciences, Jimma University, Jimma, Ethiopia

Full list of author information is available at the end of the article
}

(c) The Author(s). 2018 Open Access This article is distributed under the terms of the Creative Commons Attribution 4.0 International License (http://creativecommons.org/licenses/by/4.0/), which permits unrestricted use, distribution, and reproduction in any medium, provided you give appropriate credit to the original author(s) and the source, provide a link to the Creative Commons license, and indicate if changes were made. The Creative Commons Public Domain Dedication waiver (http://creativecommons.org/publicdomain/zero/1.0/) applies to the data made available in this article, unless otherwise stated. 


\section{Background}

Extended-spectrum $\beta$-lactamases (ESBLs) are a predominant cause of $\beta$-lactam resistance in Gram-negative bacilli (GNB) $[1,2]$. Incidences of infections caused by ESBLs producing GNB are increasing in prevalence worldwide, both in the healthcare as well as community settings, posing significant therapeutic challenges [3-5]. ESBLs are most often a plasmid mediated heterogeneous group of $\beta$-lactamase enzymes, that confer resistance to a wide range of commonly used $\beta$-lactam antibiotics including third generation cephalosporins (e.g., ceftriaxone, cefotaxime and ceftazidime) as well as monobactams (aztreonam) [6]. TEM and SHV type ESBLs used to be the dominant ESBL genotypes [7]. However, in the past decade, the CTX-M type ESBLs have become the most widely distributed and globally dominant genotypes [8].

The CTX-M type enzymes are a group of class A ESBLs that in general exhibit much higher levels of activity against cefotaxime and ceftriaxone than ceftazidime [6,9]. The presence of CTX-M type ESBLs is often associated with co-resistance phenotypes in particular to fluoroquinolones and aminoglycosides, in addition to tetracycline, and trimethoprim/sulfamethoxazole co-resistance, which is commonly observed among TEM and SHV type ESBLs $[10,11]$. The group of CTX-M type ESBLs currently constitutes more than 170 allelic variants, which cluster into five major groups based on sequence homologies. The five CTX-M groups are: CTX-M-1, CTX-M-2, CTX-M-8, CTX-M-9 and CTX-M-25 [12]. Each group consists of a number of particular variants with dominant variants being restricted in distribution to specific geographic areas, while few others are globally distributed. CTX-M-14 and CTX-M-15 were the most commonly isolated variants worldwide [10, 13].

In Africa, CTX-M-15 (of the CTX-M-1 group) is the most frequently reported variant, although some other variants were also detected in the region [14, 15]. CTX-M type ESBLs have now spread and could be detected among many different bacterial strains of clinical importance. This is particularly true for Enterobacteriaceae revealing an ESBL phenotype such as Escherichia coli and Klebsiella pneumoniae, which often cause potentially serious infections in the hospital as well as community setting [13].

In Ethiopia, multiple studies have reported prevalence of ESBLs ranging from 25 to $38.5 \%$ among Enterobacteriaceae in clinical samples obtained from various hospitals, including Jimma University Specialized Hospital (JUSH) [16-19]. However, there is no data on the prevalence and antibiotic susceptibility patterns of CTX-M type ESBLs produced by GNB. Therefore, the aim of the present study was to determine the relative frequency and distribution of the $b l a_{\text {СTX-M }}$ genes, as well as the overall susceptibility patterns in ESBL producing clinical isolates of GNB in JUSH, southwest Ethiopia.

\section{Methods \\ Study setting and clinical specimens}

A total of 224 randomly selected, non-duplicate, pure and clinically relevant Gram-negative bacilli isolates recovered from various clinical specimens submitted to the bacteriology laboratory for routine culture and antimicrobial susceptibility testing at JUSH during March to October 2014 were included in the study. The isolates were stored in $-20{ }^{\circ} \mathrm{C}$ freezers until transport and subsequently shipped to the Department of Bacteriology, Max von Pettenkofer-Institute (LMU), Munich, Germany for further screening and molecular analysis. The specimens were sent from different inpatient and outpatient units of JUSH, the only teaching and referral hospital in the southwestern part of Ethiopia, providing health services for approximately 15 million people in the catchment area. The specimens included wound swabs, urine, biopsies, sputum and others (see Additional file 1). All inpatient clinical specimens were obtained after more than $48 \mathrm{~h}$ of hospitalization of the patient. Along with the specimens, basic demographic and medical data were recorded using standard clinical and laboratory record forms.

Bacterial isolation, identification and susceptibility testing Isolation and identification of the bacterial isolates was performed using standard microbiological techniques in use at the bacteriology laboratory in JUSH [20]. At the Max von Pettenkofer-Institute (LMU), all isolates were identified to the species level by MALDI-TOF mass spectrometry (MALDI Biotyper, Bruker Daltonik, Bremen, Germany, Biotyper software package, version 3.0) [21], and then retested for antibiotic susceptibilities using VITEK $^{\circ} 2$ compact automated system (N215 and N248, bioMérieux, France), according to the instructions of the manufacturers. Software supplied by the manufacturer in compliance with the EUCAST v4.0 guidelines was used. The system included an Advanced Expert System (AES) that analysed growth patterns and detected the phenotype of organisms. Calculated MICs of piperacillin, piperacillin-tazobactam, cefotaxime, ceftazidime, cefepime, aztreonam, imipenem, meropenem, amikacin, gentamicin, ciprofloxacin, tobramycin, moxifloxacin, fosfomycin, tigecycline, colistin and trimetho$\mathrm{prim} / \mathrm{sulfamethoxazole}$ were determined and interpreted according to EUCAST v4.0 guidelines [22].

\section{ESBL screening and phenotypic tests}

All Enterobacteriaceae isolates with reduced susceptibility or resistance to ceftazidime and/or cefotaxime and/or aztreonam [23] and all non-fermenting GNB 
with multi-resistant phenotype [24] were considered as ESBL-screen positive and subjected to phenotypic and genotypic analysis. Phenotypic detection of ESBL production was performed with the VITEK $^{\circ} 2$ compact automated systems (bioMérieux, France).

\section{Detection and molecular characterization of $\beta$-lactamase genes}

Detection and molecular characterization of the $\beta$-lactamase genes was performed on all ESBL-screen positive isolates using Check-MDR CT103 Microarray Kits (Check-Points B.V., Wageningen, The Netherlands) following the manufacturer's instructions. With this assay, mutation analysis of TEM and SHV genes was performed to separate wild type (WT) alleles from ESBL variants, further AmpC $\beta$-lactamases (CMY-I/MOX, ACC, DHA, ACT/MIR, CMY-II, FOX) and carbapenemases (KPC, NDM, VIM, IMP, OXA-48-like) were investigated. Finally, CTX-M group ESBLs 1, 2, 8 plus 25, and 9 are also detected with the chip. To further define the type of CTX-M group -1 and -9 genes specifically, all positive isolates were amplified with primers suggested by Kim et al. [25]. For CTX-M-1 group, the primers with the sequence 5-cgt cacgctgttgttaggaa-3 and 5-acggctttctgccttaggtt-3 were used at $55^{\circ} \mathrm{C}$ annealing temperature to yield a $780 \mathrm{bp}$ fragment. CTX-M-9 group genes were amplified with the primers 5-tattgggagtttgagatggt-3 and 5-tccttcaactcagcaaaagt-3 at $50{ }^{\circ} \mathrm{C}$ annealing temperature to yield a $932 \mathrm{bp}$ fragment. The fragments were sequenced for allele type identification. In combination with the Check-MDR hybridization the CTX-M subtypes can thereby be identified with high confidence, although a theoretical uncertainty remains, as the gene is not completely covered by the sequencing.

\section{Quality control}

For ESBL testing, K. pneumoniae ATCC 700603 (ESBL positive), E. coli CCUG62975 (ESBL positive), E. coli ATCC 25922 (ESBL negative) and P. aeruginosa (ATCC 27853) were used as quality control (QC) in all tests.

\section{Statistical analyses}

Statistical significance for comparison of proportions was calculated by the chi-squared test using Statistical Package for Social Sciences (SPSS, version 23, SPSS, Chicago, IL, U.S.A.). A value of $P<0.05$ was considered as statistically significant.

\section{Ethical considerations}

The study was approved by Jimma University Ethical Review Board.

\section{Results}

Clinical bacterial isolates and specimens

Of the total 224 Gram-negative bacterial strains, 112 (50\%) isolates were considered as screen positive for ESBLs. These isolates consisted of 73 Enterobacteriaceae (31 Klebsiella pneumoniae, 2 Klebsiella oxytoca, 14 Enterobacter cloacae, 13 Escherichia coli, 5 Providencia stuartii, 4 Proteus mirabilis, 3 Morganella morganii, and 1 Escherichia hermanii) and 39 non-fermenting Gram-negative bacilli (14 Acinetobacter baumanii, 2 Acinetobacter pittii, 1 Acinetobacter haemolyticus, 14 Pseudomonas aeruginosa, 3 Alcaligenes faecalis, 4 Stenotrophomonas maltophilia and 1 Bordetella bronchiseptica). The majority of these isolates was recovered from inpatients $(83.9 \%, n=94)$ mainly from surgical wards (60.6\%, $n=57)$ followed by medical wards $(21.3 \%, n=$ $20)$ and from two types of specimens; wound $(54.5 \%$, $n=61)$ and urine samples $(26.8 \%, n=30)$, which together account for $81.3 \%(n=91)$ of the total isolates (see also Additional file 1). The total 112 screen positive isolates were collected from 100 patients; 90 (90\%) of patients yielded one isolate for inclusion whereas ten (10\%) patients yielded multiple species (eight patients with two species and two patients with three species).

\section{Phenotypic detection of ESBLs}

Phenotypic ESBL production was observed in $62.5 \%$ $(n=70)$ of the total screen positive isolates $(n=112)$ using VITEK $^{\ominus} 2$ compact automated system (bioMérieux, France).

\section{Genotypic detection of ESBL encoding genes}

Of the total 112 screen positive isolates, 63.4\% $(n=71)$ were positive for ESBL encoding genes by Check-MDR array. This corresponds to $91.8 \%(67 / 73)$ of the total Enterobacteriaceae and $10.3 \%(4 / 39)$ of non-fermenting Gram-negative bacilli, namely $3 P$. aeruginosa and $1 A$. faecalis isolate. No ESBL alleles were detected among Acinetobacter spp., S. maltophilia and B. bronchiseptica (Table 1). Specimen wise, $60.7 \%(n=37)$ of isolates from wound samples, $63.3 \%(n=19)$ from urine, $66.7 \%(n=8)$ from biopsy samples and all the isolates obtained from sputum samples $(n=6)$ as well as eye discharge $(n=1)$ were positive for ESBL encoding genes. Among total inpatient $(n=94)$ and outpatient $(n=18)$ isolates, ESBL genes were detected in $68.1 \%$ and $38.9 \%$ of the isolates respectively. The comparison of the difference in proportion should be taken with caution as convenient sampling was used and most specimens were obtained from inpatients. Four patients had two different ESBL-positive isolates ( $E$. cloacae and $K$. pneumoniae in two cases cases, E. coli and M. morganii, and P. aeruginosa and $A$. faecalis in one case each). One of the four patients had an SHV 238S + $240 \mathrm{~K}$ mutation bearing E. cloacae and a 
Table 1 Frequency, distribution and combinations of bla genes among screen and ESBL gene positive Gram-negative isolates

\begin{tabular}{|c|c|c|c|c|c|c|c|c|c|c|}
\hline \multirow[t]{3}{*}{ Screen positive species } & \multicolumn{2}{|c|}{ Total ESBL } & \multicolumn{8}{|c|}{ Among ESBL gene positive isolates } \\
\hline & \multirow[b]{2}{*}{ n: } & \multirow[b]{2}{*}{$\%$} & \multicolumn{2}{|c|}{ SHV E240K + G238S alone } & \multicolumn{2}{|c|}{ CTX-M alone } & \multicolumn{2}{|c|}{$\begin{array}{l}\text { CTX-M + SHV } \\
\text { E240K + G238S }\end{array}$} & \multicolumn{2}{|c|}{ Total CTXM } \\
\hline & & & $\mathrm{n}:$ & $\%$ & $\mathrm{n}:$ & $\%$ & $\mathrm{n}:$ & $\%$ & $\mathrm{n}:$ & $\%$ \\
\hline E. coli $(n=13)$ & 13 & 100 & 0 & 0 & 12 & 92.3 & 1 & 7.7 & 13 & 100 \\
\hline K. pneumoniae $(n=31)$ & 30 & 96.8 & 0 & 0 & 29 & 96.7 & 1 & 3.3 & 30 & 100 \\
\hline E. cloacae $(n=14)$ & 12 & 85.7 & 3 & 25.0 & 9 & 75.0 & 0 & 0 & 9 & 75.0 \\
\hline other Enterobacteriaceae $(n=15)$ & 12 & 80.0 & 0 & 0 & 12 & 100 & 0 & 0 & 12 & 100 \\
\hline P. aeruginosa $(n=14)$ & 3 & 21.4 & 0 & 0 & 3 & 100 & 0 & 0 & 3 & 100 \\
\hline $\begin{array}{l}\text { Other } \\
\text { Non-fermenters } \\
(n=25)\end{array}$ & 1 & 4 & 0 & 0 & 1 & 100 & 0 & 0 & 1 & 100 \\
\hline Total $(n=112)$ & 71 & 63.4 & 3 & 4.2 & 66 & 92.9 & 2 & 2.8 & 68 & 95.8 \\
\hline
\end{tabular}

includes 3 M. morganii, 4 P. mirablis, 5 P. stuartii, 2 K. oxytoca and 1 E. hermanii

$\mathrm{b}_{\text {includes }} 17$ Acinetobacter species (14 A. baumanii, 2 A. pittii and 1 A. haemolyticus), 3 A. faecalis, 4 S. maltophilia and 1 B. bronchiseptica

CTX-M-15 positive $K$. pneumoniae in the specimen, whereas the three other patients each had two different species each positive for CTX-M-15.

\section{Frequency and distribution of bla}

From a total of 71 isolates carrying ESBL encoding genes, 68 (95.8\%) carried CTX-M genes either alone or in combination with SHV and/or TEM genes. Sixty-four out of 67 (95.5\%) Enterobacteriaceae and all non-fermenting GNB $(n=4)$ which carried ESBL encoding genes, were positive for CTX-M genes. The remaining three isolates negative for CTX-M (4.2\%) carried SHV-type ESBLs $(\mathrm{G} 238 \mathrm{~S}+\mathrm{E} 240 \mathrm{~K})$ genes and were found to be $E$. cloacae obtained from wound samples. All TEM and SHV $\beta$-lactam genes detected were wild type except five G238S + E240K SHV type ESBLs. Three of the five were detected in E. cloacae in combination with wild type TEM. The other two were found in one E. coli and $K$. pneumoniae isolate along with CTX-M genes (Table 1).

\section{Combinations of bla $a_{\text {СтX-M }}$ with other $\beta$-lactamase genes}

Multiple $\beta$-lactamase genes in a single strain were observed in $83.1 \%(n=59)$ of the total isolates carrying ESBL encoding genes. From a total of 68 CTX-M positive isolates, 12 (17.6\%) harbored CTX-M alone. The remaining $56(82.4 \%)$ isolates carried CTX-M in combination with wild type TEM and/or SHV (except two SHV E240K + G238S) in different frequencies, which is partly explained due to the general presence of $\beta$-lactamases in some strains e.g. in Klebsiella spp. (Table 1).

\section{Frequency and distribution of CTX-M groups and types} CTX-M group 1 was the most dominant CTX-M group detected in 66 of 68 CTX-M positive isolates (97.1\%), either alone $(n=63,92.6 \%)$ or in combination with other groups $(n=3,4.5 \%)$. All CTX-M-1 genes were sequenced and all were found to be allele CTX-M-15.
The remaining two (2.9\%) CTX-M positive isolates carried CTX-M group 9 (Table 2) genes which upon sequencing were identified as allele CTX-M-24.

\section{Antibiotic susceptibility pattern of CTX-M positive gram- negative bacilli isolates}

The antibiotic susceptibility testing for CTX-M-positive Enterobacteriaceae isolates demonstrated a MIC in the respective susceptible range in $<2 \%$ of cases against cephalosporins according to EUCAST guidelines. Susceptibilities to carbapenems and a few other substances were found to be much higher. In terms of non-susceptibility, the highest level of antibiotic resistances was observed as expected against $\beta$-lactams such as piperacillin and cephalosporins, but also against trimethoprim-sulfamethoxazole (92.2\%), gentamicin (89.1\%), and quinolones (75\%). No isolates showed full resistance to imipenem or meropenem, and only $3.1 \%$ and $1.6 \%$ tested intermediate for these substances, respectively (Table 3 ). One E. coli isolate tested positive for CTX-M-15 but was measured susceptible to third generation cephalosporins using VITEK 2 as well as disc diffusion tests.

\section{Co-resistance (co-non-susceptibility) to non- $\beta$-lactam antibiotics}

All the CTX-M-positive Enterobacteriaceae $(n=64,100 \%)$ and $P$. aeruginosa $(n=3,100 \%)$ were non-susceptible to $\geq 1$ agent in $\geq 3$ antimicrobial categories and hence defined as multidrug resistant (MDR) according to the international expert proposal for interim standard definitions for acquired resistance promoted by the European Centre for Disease Prevention and Control (ECDC) [26]. About 92.2\%, 78.1\% and $92.2 \%$ of the total CTX-M-positive Enterobacteriaceae were found to be non-susceptible (co-resistant) to aminoglycosides, fluoroquinolones and trimethoprim-sulfamethoxazole, respectively (Fig. 1). 
Table 2 Frequency and distribution of CTX-M groups among CTX-M positive Gram-negative bacilli isolates

\begin{tabular}{|c|c|c|c|c|c|c|c|c|c|c|c|c|c|c|c|c|c|c|}
\hline \multirow[t]{3}{*}{ CTX-M positive species } & \multicolumn{8}{|c|}{ CTX-M groups (total) } & \multicolumn{10}{|c|}{ CTX-M group combinations } \\
\hline & \multicolumn{2}{|c|}{ CTX-M-1 } & \multicolumn{2}{|c|}{ CTX-M-2 } & \multicolumn{2}{|c|}{ CTX-M-8 + 25} & \multicolumn{2}{|c|}{$\overline{\text { CTX-M-9 }}$} & \multicolumn{2}{|c|}{ CTX-M-1 alone } & \multicolumn{2}{|c|}{ CTX-M-1 + 2} & \multicolumn{2}{|c|}{ CTX-M-1 + 9} & \multicolumn{2}{|c|}{$\begin{array}{l}\text { CTX-M-1 } \\
+2+8+25 \\
\end{array}$} & \multicolumn{2}{|c|}{ CTX-M-9 alone } \\
\hline & $\mathrm{n}:$ & $\%$ & $\mathrm{n}:$ & $\%$ & $\mathrm{n}:$ & $\%$ & $\mathrm{n}:$ & $\%$ & $\mathrm{n}:$ & $\%$ & $\mathrm{n}:$ & $\%$ & $\mathrm{n}:$ & $\%$ & $\mathrm{n}:$ & $\%$ & $\mathrm{n}:$ & $\%$ \\
\hline E. coli $(n=13)$ & 12 & 92.3 & 0 & 0 & 0 & 0 & 2 & 15.4 & 11 & 84.6 & 0 & 0 & 1 & 7.7 & 0 & 0 & 1 & 7.7 \\
\hline K. pneumoniae $(n=30)$ & 30 & 100 & 1 & 3.3 & 0 & 0 & 0 & 0 & 29 & 96.7 & 1 & 3.3 & 0 & 0 & 0 & 0 & 0 & 0 \\
\hline E. cloacae $(n=9)$ & 9 & 100 & 0 & 0 & 0 & 0 & 0 & 0 & 9 & 100 & 0 & 0 & 0 & 0 & 0 & 0 & 0 & 0 \\
\hline $\begin{array}{l}\text { Other Enterobacteriaceae }{ }^{a} \\
(n=12)\end{array}$ & 11 & 91.7 & 0 & 0 & 0 & 0 & 1 & 8.3 & 11 & 91.7 & 0 & 0 & 0 & 0 & 0 & 0 & 1 & 8.3 \\
\hline P. aeruginosa $(n=3)$ & 3 & 100 & 1 & 33.3 & 1 & 33.3 & 0 & 0 & 2 & 66.7 & 0 & 0 & 0 & 0 & 1 & 33.3 & 0 & 0 \\
\hline $\begin{array}{l}\text { Other Non-Fermenters }{ }^{\mathrm{b}} \\
(n=1)\end{array}$ & 1 & 100 & 0 & 0 & 0 & 0 & 0 & 0 & 1 & 100 & 0 & 0 & 0 & 0 & 0 & 0 & 0 & 0 \\
\hline Total $(n=68)$ & 66 & 97.1 & 2 & 2.9 & 1 & 1.5 & 3 & 4.4 & 63 & 92.6 & 1 & 1.5 & 1 & 1.5 & 1 & 1.5 & 2 & 2.9 \\
\hline
\end{tabular}

includes 3 M. morganii, 4 P. mirablis, 2 P. stuartii, 2 K. oxytoca and 1 E. hermani

$\mathrm{b}_{\text {includes } 1} \mathrm{~A}$. faecalis

\section{Non-susceptibility pattern in CTX-M and non-CTX-M carrying isolates}

Both CTX-M $(n=64)$ and non-CTX-M-producing $(n=119)$ Enterobacteriaceae isolates have comparable non-susceptibility patterns to piperacillin/tazobactam, imipenem, meropenem, fosfomycin, and colistin/polymyxin $\mathrm{B}(P>0.05)$. However, the non-susceptibility rate to all other antibiotics tested were all significantly higher among CTX-M-positive isolates compared to non-CTX-M ESBL-carrying isolates $(P<0.001)$ (Fig. 2). All the CTX-M negative isolates were also non-ESBLs except for three isolates expressing SHV type ESBLs. Unlike seen with CTX-M ESBLs, this did not affect the other non-susceptibilities.

\section{Discussion}

The present study is the first report describing the molecular epidemiology of ESBL-encoding genes in Ethiopia. We demonstrate a high level of prevalence of CTX-M-type ESBLs among all ESBL positive isolates at JUSH. In total, $95.8 \%$ of all ESBL genes detected were of CTX-M type, and almost unanimously CTX-M-1 group variant type 15 (97.1\% of all CTX-M positive isolates). These findings are in accordance with the fact that the CTX-M type ESBLs are the most widely distributed and globally dominant ESBL genotypes to date [13, 27, 28]. Of the groups, CTX-M-1 was also described to be highly prevalent in Italy [29], India [30], Switzerland [31], Saudi-Arabia [32], Syria [33], Pakistan [34] and China [35].

Table 3 In vitro antimicrobial resistance pattern of CTX-M-positive Gram-negative isolates

\begin{tabular}{|c|c|c|c|c|c|c|c|c|c|c|c|c|c|c|c|c|c|}
\hline \multirow[t]{2}{*}{ Species } & \multicolumn{17}{|c|}{ CTX-M positive isolate $\%$ resistance } \\
\hline & $\mathrm{PI}$ & PIT & CTX & CAZ & CPM & AT & IMP & MRP & AK & HLG & TOB & CIP & MOX & $\mathrm{FO}$ & TGC & $\mathrm{CL}$ & $\mathrm{COT}$ \\
\hline E. coli $(n=13)$ & 100 & 30.8 & 92.3 & 92.3 & 92.3 & 92.3 & 0 & 0 & 7.7 & 76.9 & 76.9 & 92.3 & 84.6 & 7.7 & 0 & 7.7 & 84.6 \\
\hline K. pneumoniae $(n=30)$ & 100 & 60 & 100 & 96.7 & 96.7 & 96.7 & 0 & 0 & 0 & 90 & 96.7 & 66.7 & 80 & 0 & 0 & 0 & 93.3 \\
\hline E. cloacae $(n=9)$ & 100 & 0 & 100 & 100 & 100 & 100 & 0 & 0 & 0 & 88.9 & 88.9 & 22.2 & 77.8 & 0 & 0 & 0 & 100 \\
\hline M. morganii $(n=3)$ & 100 & 0 & 100 & 100 & 100 & 100 & 0 & 0 & 0 & 100 & 100 & 66.7 & 100 & 100 & $\mathbb{R}$ & $\mathbb{I R}$ & 100 \\
\hline P. mirablis $(n=4)$ & 100 & 0 & 100 & 100 & 100 & 100 & 0 & 0 & 0 & 100 & 100 & 25 & 25 & 50 & $\mathbb{R}$ & $\mathbb{I R}$ & 75 \\
\hline P. stuartii $(n=2)$ & 100 & 0 & 100 & 100 & 100 & 100 & 0 & 0 & 0 & $\mathbb{R}$ & $\mathbb{R}$ & 50 & 50 & 50 & $\mathbb{R}$ & $\mathbb{R}$ & 100 \\
\hline K. oxytoca $(n=2)$ & 100 & 50 & 100 & 100 & 100 & 100 & 0 & 0 & 0 & 100 & 100 & 0 & 50 & 0 & 0 & 0 & 100 \\
\hline E. hermanii $(n=1)$ & $\mathrm{R}$ & $\mathrm{R}$ & $\mathrm{R}$ & $\mathrm{R}$ & $\mathrm{R}$ & $\mathrm{R}$ & S & $S$ & $\mathrm{~s}$ & $\mathrm{R}$ & $\mathrm{R}$ & $\mathrm{R}$ & $\mathrm{R}$ & S & $S$ & S & $\mathrm{R}$ \\
\hline $\begin{array}{l}\text { Total Enterobacteriaceae } \\
(n=64)\end{array}$ & 100 & 35.9 & 98.4 & 96.9 & 96.9 & 96.9 & 0 & 0 & 1.6 & 89.1 & 92.2 & 59.4 & 75 & 10.9 & 14.1 & 15.6 & 92.2 \\
\hline P. aeruginosa $(n=3)$ & 66.7 & 66.7 & $\mathbb{R}$ & 33.3 & 66.7 & 66.7 & 0 & 0 & 33.3 & 66.7 & 66.7 & 100 & 100 & 100 & $\mathbb{R}$ & 0 & $\mathbb{R}$ \\
\hline A. faecalis $(n=1)$ & $\mathrm{R}$ & $S$ & $\mathrm{IE}$ & $\mathrm{IE}$ & $\mathrm{IE}$ & $\mathrm{R}$ & $S$ & $S$ & $S$ & $S$ & S & $\mathrm{IE}$ & $\mathrm{s}$ & $\mathrm{IE}$ & $\mathrm{IE}$ & $\mathrm{IE}$ & $\mathrm{IE}$ \\
\hline Total GNB $(n=68)$ & 98.5 & 36.8 & 97.1 & 92.6 & 94.1 & 95.6 & 0 & 0 & 2.9 & 88.2 & 91.2 & 60.3 & 76.5 & 14.7 & 17.6 & 14.7 & 91.2 \\
\hline
\end{tabular}

Key: PI piperacillin, PIT piperacillin/tazobactam, CTX cefotaxime, CAZ ceftazidime, CPM cefepime, AT aztreonam, IMP imipenem, MRP meropenem, AK amikacin, HLG gentamicin, TOB tobramycin, CIP ciprofloxacin, MOX moxifloxacin, FO fosfomycine, TGC tigecycline, CL colistin, COT trimethoprim/sulfamethoxazole $n$ number of isolates, $S$ sensitive, $R$ resistant, $I R$ intrinsic resistance, $I E$ insufficient evidence 


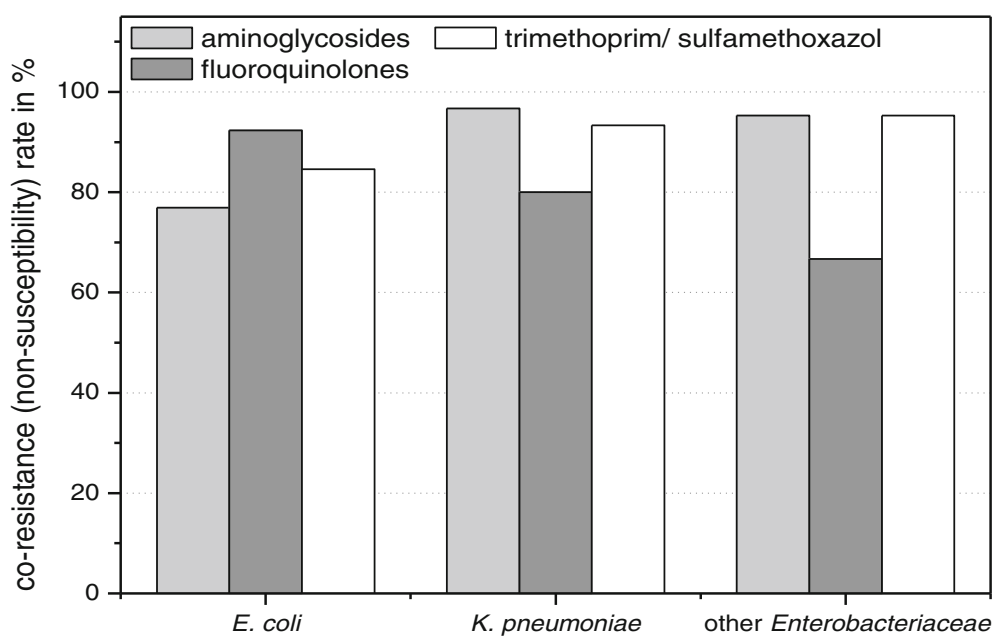

Fig. 1 Bar graph showing the non-susceptibility pattern of the CTX-M positive E. coli $(n=13)$, K. pneumoniae $(n=30)$ and other Enterobacteriaceae $(n=21)$ against aminoglycosides, fluoroquinolones and trimethoprim-sulfamethoxazole

Factors and mechanisms which contribute to the emergence and increasing prevalence of CTX-M ESBLs of all groups are complex and may involve both, plasmid dissemination as well as clonal spread of bacterial strains $[36,37]$. In addition, the selective pressure exerted by the frequent use of wide spectrum cephalosporins may promote their epidemiological success [10, 28, 38]. Especially in Ethiopia, the widespread misuse and overuse of cephalosporins may contribute to the selection and spread of CTX-M gene carrying clones [39-41]. The frequency of the CTX-M genotype among the ESBL gene-positive $E n$ terobacteriaceae isolates was also remarkably high (95.5\%) compared to similar findings among clinical Enterobacteriaceae isolates with prevalence rates of 91\% in Brazil [42], 80.3\% in Germany [43] and 79\% in Switzerland [31].

Other than E. coli (92.3\% CTX-M-15) and K. pneumoniae (100\% CTX-M-15), CTX-M were also detected among other members of ESBL producing Enterobacteriaceae (K. oxytoca, M. morganii, P. mirablis, P. stuartii, E. hermannii and E. cloacae) as well as non-fermenting GNB (P. aeruginosa and A. fecalis) in $87.5 \%(n=21)$ and $100 \%$ $(n=4)$, respectively. Out of all screen positive isolates (112) 41 were found to be non-ESBL producers. Thereby, most (35/41) were lactose non-fermenting GNB with

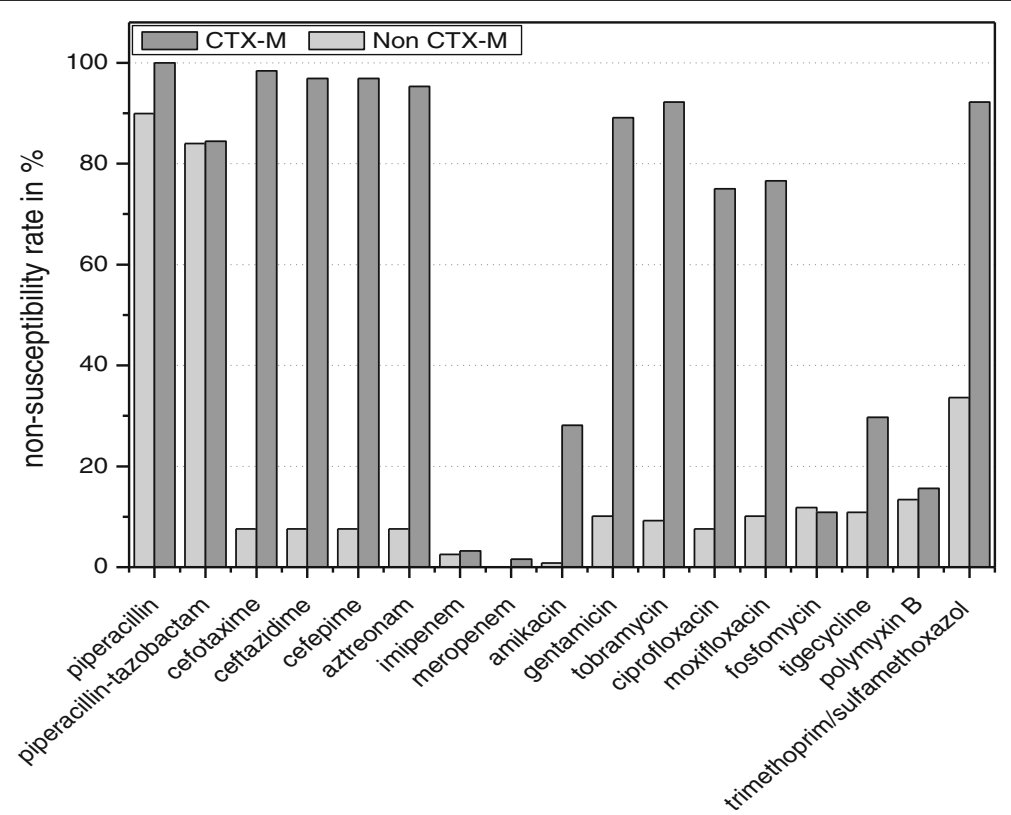

Fig. 2 Comparison of non-susceptibility patterns of bla $a_{\mathrm{CTX}-\mathrm{M}}(n=64)$ and non-bla $a_{\mathrm{CTX}-\mathrm{M}}(n=119)$ Enterobacteriaceae isolates against the 17 different antibiotics tested 
known extensive intrinsic resistance mechanisms. Other isolates may be resistant due to genes not tested within this study, or due to derepression of wild type $\beta$-lactamases or even permeability defects. Among screen positive Enterobacteriaceae isolates, 92\% (67/73) were also positive for an ESBL gene tested within this study.

Although, this study was small, it indicates the dissemination of the CTX-M genes to other GNB besides Enterobacteriaceae in Jimma. Similar findings have been reported in Switzerland [31], Argentina [44], Netherlands [45] and Japan [13]. The frequency of ESBL gene positive Pseudomonas aeruginosa was low $(21.4 \%, n=3)$ when compared to other GNB. This is probably due to the fact that most resistance mechanisms in Pseudomonas aeruginosa are mediated by the overproduction of AmpC $\beta$-lactamases as well as acquired metallo- $\beta$-lactamases, decreased permeability and efflux pumps [46]. In addition, plasmid incompatibility and host range of ESBL encoding plasmids might also play a role in our setting [13]. The emergence and spread of CTX-M-producing isolates in the community, particularly among $E$. coli in urinary tract infections (UTI), were reported from China [47], Brazil [48] and the UK [49]. A trend in this direction can also be seen in our study, as all the outpatient urine isolates of $E$. coli $(n=2)$, K. pneumoniae $(n=2), M$. morganii $(n=1), P$. mirablis $(n=1)$ and E. cloacae $(n=1)$ with an ESBL gene were shown to carry CTX-M genes. However, the total sample size of outpatient isolates in the present study is small compared to the inpatient sample number.

The overall resistance pattern of the total CTX-M positive Enterobacteriaceae is very high for most antibiotics tested in the present study. The carbapenems $(0 \%$ resistance) followed by amikacin (3\% resistance) were found to have the highest susceptibility rates. However, all CTX-M-positive isolates identified in this study showed a MDR phenotype as well as remarkably high rates of co-resistance to fluoroquinolones, aminoglycosides, and trimethoprim/sulfamethoxazole. Only one $E$. coli isolate positive for an ESBL gene (CTX-M-15) was not resistant against third generation cephalosporins, while still maintaining an MDR phenotype. In this particular case, the CTX-M operon seems to be non-functional perhaps due to mutations. These findings are consistent with studies from Ghana [50], Lebanon [51] and India [52] which propose imipenem and amikacin as possible drugs for the management of infection caused by CTX-M-producing isolates. The results are also in accordance with findings of high prevalence of MDR phenotype (88.4\%) among ESBL-producing E. coli and K. pneumoniae isolates in a previous phenotypic characterization of strains in JUSH [17]. Comparably high rates of co-resistance to non- $\beta$-lactam antibiotics were also reported from Brazil [42], South Korea [53] and Indian hospitals [54].
Surprisingly, colistin/polymyxin, which is not available in Ethiopia, showed resistance rates of above $10 \%$. However, this rate has to be interpreted with caution, as the data based on VITEK 2 testing system is unreliable for detecting colistin resistance [55], and results obtained by these methods may be overrated and require confirmation by ISO-standard broth microdilution method as nowadays recommended by EUCAST [56, 57]. As the respective recommendation was issued after completion of the study, it was not taken into consideration.

In the present study, only clinically relevant isolates of in- and outpatients were used, a screening upon admission, or screening of healthy controls was not performed. However, the high rates of ESBL positive organisms in outpatients without contact to the health care system within the last 3 months, argues for considerable ESBL carrier rates among the general population. Within the study population, mainly samples from internal medicine, pediatrics and ICU were ESBL positive and MDR, whereas in the surgical patient group many patients were found to harbor non-fermenters with MDR phenotype which are negative for the ESBL and carbapenemase genes tested within this study (see Additional file 2).

This conclusion is supported by a study conducted at black lion hospital in Addis Ababa (Ethiopia) reporting a high gastrointestinal colonization rate with ESBL producing Enterobacteriaceae among hospitalized patients [58]. It is well known, that many of the patients who develop health care-associated ESBL infections have preceding colonization of the gastrointestinal tract [59,60]. A combination based on lack of hygiene and high colonization rates with ESBL positive organisms are likely to drive the ESBL rates in JUSH.

Within the sample group, other prominent resistance determinants were also investigated as part of the CT103 panel. Thereby, no KPC, NDM-1, VIM, IMP or Oxa48-like coding organism was detected. Previously, we could demonstrate the presence of NDM-1 in Acinetobacter baumannii in the area [61]. NDM-1 gene transfer to other isolates seems not to have occurred in relevant numbers. However, the presence of CTX-M-15 genes in different species in such high prevalence argues for horizontal gene transfer currently or in the past. The transfer might have occurred by plasmid exchange, which is especially common among Enterobacteriaceae, or by less frequent recombination events, e.g. involving IS elements. How recent or frequent such events have been cannot be elucidated given the methodology used, as the genes are found in numerous different species and isolates, it certainly cannot be explained simply by local clonal expansion of one strain.

\section{Conclusions}

This study demonstrates a remarkably high level of CTX-M genes in GNB isolated in JUSH. The most 
predominant group was CTX-M-1 allele 15 and a few percent CTX-M-9 allele 24 among all the ESBLs gene positive clinical isolates. In South Africa, CTX-M-2 and -3 group are most prevalent, and CTX-M-14 and -15 in Egypt [62]. Meropenem, imipenem, colistin and amikacin were found to have the highest in vitro efficacy against the CTX-M-producing isolates. The high level of resistance to $\beta$-lactam and non- $\beta$-lactam antibiotics as well as the trend of a MDR profile associated with the CTX-M genes are alarming and emphasize the need for diagnostic antimicrobial susceptibility testing for appropriate choice of antimicrobial therapy and limiting the spread of antimicrobial resistance in Ethiopia and in the region.

\section{Additional files}

Additional file 1: Distribution and frequency of GNB isolates in different clinical specimens. (PPTX $68 \mathrm{~kb}$ )

Additional file 2: Rates of ESBL and MDR in view of different hospital departments. (PPTX $44 \mathrm{~kb}$ )

\section{Acknowledgements}

We thank Gabriele Liegl for excellent technical assistance as well as Dr. A.-C. Neumann for excellent support with figure generation.

\section{Funding}

The study was supported by Jimma University (to AZ and AW) and the German Center for Infection Research (DZIF) (AW, MH) as well as the Else Kröner-Fresenius-Stiftung (EKFS) to AW.

\section{Availability of data and materials}

All data generated or analyzed during this study are included in this published article [and its supplementary information files].

\section{Authors' contributions \\ $A Z, S S, T B$ and $A W$ designed the study and protocol. AZ, MP, GL, MH and AW performed laboratory work. AZ, MP, SS, MH, TB, MM and AW analyzed the data and wrote the manuscript. All authors read and approved the final manuscript.}

\section{Ethics approval and consent to participate}

The study was approved by Jimma University Ethical Review Board. Bacterial isolates were anonymized and re-analyzed in LMU Munich (Germany) as purified bacterial strains. For such analysis, no ethical clearance is required at LMU Munich.

\section{Consent for publication}

Not applicable.

\section{Competing interests}

The authors declare that they have no competing interests.

\section{Publisher's Note}

Springer Nature remains neutral with regard to jurisdictional claims in published maps and institutional affiliations.

\section{Author details}

${ }^{1}$ Chair of Medical Microbiology and Hospital Epidemiology, Max von Pettenkofer Institute, Faculty of Medicine, LMU Munich, Marchioninistr. 17 81377 Munich, Germany. ${ }^{2}$ Institute of Health Sciences, Jimma University, Jimma, Ethiopia. ${ }^{3}$ Center for International Health $(\mathrm{ClH})$, University of Munich (LMU), 80802 Munich, Germany. ${ }^{4}$ Division of Infectious Diseases and Tropical Medicine, Medical Center of the University of Munich (LMU), 80802 Munich,
Germany. ${ }^{5}$ German Center for Infection Research (DZIF), Partner Site Munich, 80802 Munich, Germany. ${ }^{6}$ Plant Genome and Systems Biology, Helmholtz Center Munich, German Research Center for Environmental Health, 85764 Neuherberg, Germany.

Received: 1 March 2018 Accepted: 5 October 2018

Published online: 20 October 2018

\section{References}

1. Ruppé É, Woerther PL, Barbier F. Mechanisms of antimicrobial resistance in gram-negative bacilli. Ann Intensive Care. 2015:5(1):1.

2. Sutton SS. What are extended-spectrum beta-lactamases? JAAPA. 2014;27(3): $14-7$

3. Chong Y, Shimoda S, Yakushiji H, Ito Y, Miyamoto T, Kamimura T, et al. Community spread of extended-spectrum $\beta$-lactamase-producing Escherichia coli, Klebsiella pneumoniae and Proteus mirabilis: a long-term study in Japan. J Med Microbiol. 2013;62(7):1038-43.

4. Kassakian SZ, Mermel LA. Changing epidemiology of infections due to extended spectrum beta-lactamase producing bacteria. Antimicrob Resist Infect Control. 2014:3(1):9.

5. WHO. Antimicrobial resistance: global report on surveillance. 2014. http:// www.who.int/drugresistance/documents/surveillancereport/en/. Accessed 17 Feb 2018.

6. Ghafourian S, Sadeghifard N, Soheili S, Sekawi Z. Extended spectrum betalactamases: definition, classification and epidemiology. Curr Issues Mol Biol. 2015;17:11-21.

7. Thenmozhi S, Moorthy K, Sureshkumar B, Suresh M. Antibiotic resistance mechanism of ESBL producing Enterobacteriaceae in clinical field: a review. Int J Pure Appl Biosci. 2014;2(3):207-26.

8. Adamski CJ, Cardenas AM, Brown NG, Horton LB, Sankaran B, Prasad BV, et al. Molecular basis for the catalytic specificity of the CTX-M extendedspectrum $\beta$-lactamases. Biochemistry. 2014;54(2):447-57.

9. Shaikh S, Fatima J, Shakil S, Rizvi SMD, Kamal MA. Antibiotic resistance and extended spectrum beta-lactamases: types, epidemiology and treatment. Saudi J Biol Sci. 2015;22(1):90-101.

10. Lahlaoui $H$, Khalifa ABH, Moussa MB. Epidemiology of Enterobacteriaceae producing CTX-M type extended spectrum $\beta$-lactamase (ESBL). Med Mal Infect. 2014:44(9):400-4.

11. Östholm Balkhed $\AA$, Tärnberg M, Monstein HJ, Hällgren A, Hanberger H, Nilsson LE. High frequency of co-resistance in CTX-M-producing Escherichia coli to non-beta-lactam antibiotics, with the exceptions of amikacin, nitrofurantoin, colistin, tigecycline, and fosfomycin, in a county of Sweden. Scand J Infect Dis. 2013;45(4):271-8.

12. Bush K, Palzkill T, Jacoby G. ß-lactamase classification and amino acid sequences for TEM, SHV and OXA extended-Spectrum and inhibitor resistant enzymes: Lahey Clinic; 2015. https://www.lahey.org/Studies/. Accessed 20 Feb 2018

13. Zhao WH, Hu ZQ. Epidemiology and genetics of CTX-M extendedspectrum $\beta$-lactamases in gram-negative bacteria. Crit Rev Microbiol. 2013:39(1):79-101.

14. Storberg V. ESBL-producing Enterobacteriaceae in Africa-a non-systematic literature review of research published 2008-2012. Infect Ecol Epidemiol. 2014:4. https://doi.org/10.3402/iee.v4.20342

15. Ehlers MM, Veldsman C, Makgotlho EP, Dove MG, Hoosen AA, Kock MM Detection of blaSHV, blaTEM and blaCTX-M antibiotic resistance genes in randomly selected bacterial pathogens from the Steve Biko academic hospital. FEMS Immunol Med Microbiol. 2009;56(3):191-6.

16. Mulisa G, Selassie L, Jarso G, Shiferew T, Zewdu A. Prevalence of extended Spectrum Beta-lactamase producing Enterobacteriaceae: a cross sectional study at Adama hospital, Adama, Ethiopia. J Emerg Infect Dis. 2016;1(102):2.

17. Siraj SM, Ali S, Wondafrash B. Extended-spectrum-lactamase production and antimicrobial resistance in Klebsiella pneumoniae and Escherichia coli among inpatients and outpatients of Jimma University specialized hospital, south-west, Ethiopia. Afr J Microbiol Res. 2014;8(43):3687-94.

18. Seid J, Asrat D. Occurrence of extended spectrum $\beta$-lactamase enzymes in clinical isolates of Klebsiella species from Harar region, eastern Ethiopia. Acta Trop. 2005;95(2):143-8

19. Mulualem Y, Kasa T, Mekonnen Z, Suleman S. Occurrence of extended spectrum beta lactamases in multi-drug resistant Escherichia coli isolated from a clinical setting in Jimma University specialized hospital, Jimma, southwest Ethiopia. East Afr J Public Health. 2012;9(2):58-61. 
20. Vandepitte J, Verhaegen J, Engbaek K, Rohner P, Piot P, Heuck C. Basic laboratory procedures in clinical bacteriology: World Health Organization; 2003. http://apps.who.int/iris/bitstream/10665/42696/1/9241545453.pdf. Accessed 20 Feb 2018

21. Wieser A, Schneider L, Jung J, Schubert S. MALDI-TOF MS in microbiological diagnostics -identification of microorganisms and beyond (mini review) Appl Microbiol Biotechnol. 2012;93(3):965-74.

22. European Committee on Antimicrobial Susceptibility Testing. Breakpoint tables for interpretation of MICs and zone diameters. Version 4.0, valid from 2014-01-01. EUCAST. https://asmsig.files.wordpress.com/2014/11/ breakpoint_table_v_4-01-2014.pdf. Accessed 20 Feb 2018.

23. European Committee on Antimicrobial Susceptibility Testing. EUCAST guidelines for detection of resistance mechanisms and specific resistances of clinical and/or epidemiological importance. Version 1.0: EUCAST; 2013. http://www.eucast.org/fileadmin/src/media/PDFs/EUCAST_files/Resistance_ mechanisms/EUCAST_detection_of_resistance_mechanisms_v1.0_20131211. pdf. Accessed 20 Feb 2018

24. Dally S, Lemuth K, Kaase M, Rupp S, Knabbe C, Weile J. DNA microarray for genotyping antibiotic resistance determinants in Acinetobacter baumannii clinical isolates. Antimicrob Agents Chemother. 2013;57(10):4761-8.

25. Kim J, Lim YM, Jeong YS, Seol SY. Occurrence of CTX-M-3, CTX-M-15, CTX$\mathrm{M}-14$, and CTX-M-9 extended-spectrum beta-lactamases in Enterobacteriaceae clinical isolates in Korea. Antimicrob Agents Chemother. 2005:49(4):1572-5

26. Magiorakos AP, Srinivasan A, Carey R, Carmeli Y, Falagas M, Giske C, et al. Multidrug-resistant, extensively drug-resistant and pandrug-resistant bacteria: an international expert proposal for interim standard definitions for acquired resistance. Clin Microbiol Infect. 2012:18(3):268-81.

27. Canton R, Coque TM. The CTX-M beta-lactamase pandemic. Curr Opin Microbiol. 2006;9(5):466-75.

28. Rossolini GM, D'Andrea MM, Mugnaioli C. The spread of CTX-M-type extended-spectrum beta-lactamases. Clin Microbiol Infect. 2008;14(Suppl 1):33-41.

29. Mugnaioli C, Luzzaro F, De Luca F, Brigante G, Perilli M, Amicosante G, et al. CTX-M-type extended-spectrum $\beta$-lactamases in Italy: molecular epidemiology of an emerging countrywide problem. Antimicrob Agents Chemother. 2006;50(8):2700-6.

30. Singh A, Shahid M, Sobia F, Khan HM. Occurrence and molecular epidemiology of Bla CTX-M, including co-occurrence of bla TEM and Bla SHV genes, and sul1 association in Indian Enterobacteriaceae. Int J Antimicrob Agents. 2012;39(2):184-5.

31. Lartigue MF, Zinsius C, Wenger A, Bille J, Poirel L, Nordmann P. Extendedspectrum $\beta$-lactamases of the CTX-M type now in Switzerland. Antimicrob Agents Chemother. 2007;51(8):2855-60

32. Al-Agamy MH, Shibl AM, Hafez MM, Al-Ahdal MN, Memish ZA, Khubnani H. Molecular characteristics of extended-spectrum $\beta$-lactamase-producing Escherichia coli in Riyadh: emergence of CTX-M-15-producing E. coli ST131. Ann Clin Microbiol Antimicrob. 2014;13(1):1.

33. Ibrahim AS, Youssef N. Prevalence of CTX-M, TEM and SHV Betalactamases in clinical isolates of Escherichia Coli and Klebsiella Pneumoniae isolated from Aleppo University Hospitals, Aleppo, Syria. Arch Clin Infect Dis. 2015;10(2):e22540.

34. Khan E, Schneiders T, Zafar A, Aziz E, Parekh A, Hasan R. Emergence of CTXM Group 1-ESBL producing Klebsiella pneumonia from a tertiary care Centre in Karachi, Pakistan. J Infect Dev Ctries. 2010;4(08):472-6.

35. Shi H, Sun F, Chen J, Ou Q, Feng W, Yong X, Xia P. Epidemiology of CTX-Mtype extended-spectrum beta-lactamase (ESBL)-producing nosocomialEscherichia coli infection in China. Ann Clin Microbiol Antimicrob. 2015; 14(1):1.

36. Cantón R, González-Alba JM, Galán JC. CTX-M enzymes: origin and diffusion. Front Microbiol. 2012;3:110

37. D'Andrea MM, Arena F, Pallecchi L, Rossolini GM. CTX-M-type $\beta$-lactamases: a successful story of antibiotic resistance. Int J Med Microbiol. 2013;303(6): 305-17.

38. Gelband H, Miller-Petrie M, Pant S, Gandra S, Levinson J, Barter D, et al. State of the World's Antibiotics 2015: Center for Disease Dynamics, Economics \& Policy; 2015. https://www.cddep.org/wp-content/uploads/2017/06/swa_ edits_9.16.pdf. Accessed 20 Feb 2018

39. Shimels T, Bilal Al, Mulugeta A. Evaluation of ceftriaxone utilization in internal medicine wards of general hospitals in Addis Ababa, Ethiopia: a comparative retrospective study. J Pharm Policy Pract. 2015;8(1):1.
40. Worku S, G/Mariam A. Practice of self medication in Jimma town. Ethiop J Health Dev 2003:17:111-116.

41. Ayinalem GA, Gelaw BK, Belay AZ, Linjesa JL. Drug use evaluation of ceftriaxone in medical ward of Dessie referral hospital, north East Ethiopia. Int J Basic Clin Pharmacol. 2013;2(6):711-7.

42. Seki LM, Pereira PS, de Souza Conceição M, Souza MJ, Marques EA, Carballido JM, et al. Molecular epidemiology of CTX-M producing Enterobacteriaceae isolated from bloodstream infections in Rio de Janeiro, Brazil: emergence of CTX-M-15. Braz J Infect Dis. 2013;17(6):640-6.

43. Schmiedel J, Falgenhauer L, Domann E, Bauerfeind R, Prenger-Berninghoff $E$, Imirzalioglu C, Chakraborty T. Multiresistant extended-spectrum $\beta$ lactamase-producing Enterobacteriaceae from humans, companion animals and horses in Central Hesse, Germany. BMC Microbiol. 2014;14(1):1.

44. Quinteros M, Radice M, Gardella N, Rodriguez M, Costa N, Korbenfeld D, et al. Extended-spectrum $\beta$-lactamases in Enterobacteriaceae in Buenos Aires, Argentina, public hospitals. Antimicrob Agents Chemother. 2003; 47(9):2864-7.

45. Al Naiemi N, Bart A, De Jong M, Vandenbroucke-Grauls C, Rietra P, DebetsOssenkopp Y. Widely distributed and predominant CTX-M extendedspectrum $\beta$-lactamases in Amsterdam, the Netherlands. J Clin Microbiol. 2006:44(8):3012-4.

46. Noyal M, Menezes G, Harish B, Sujatha S, Parija S. Simple screening tests for detection of carbapenemases in clinical isolates of nonfermentative gramnegative bacteria. Indian J Med Res. 2009;129(6):707-12.

47. Xia S, Fan X, Huang Z, Xia L, Xiao M, Chen R, et al. Dominance of CTX-Mtype extended-spectrum $\beta$-lactamase (ESBL)-producing Escherichia coli isolated from patients with community-onset and hospital-onset infection in China. PLoS One. 2014;9(7):e100707.

48. Minarini LA, Poirel L, Trevisani NA, Darini ALC, Nordmann P. Predominance of CTX-M-type extended-spectrum $\beta$-lactamase genes among enterobacterial isolates from outpatients in Brazil. Diagn Microbiol Infect Dis. 2009:65(2):202-6.

49. Woodford N, Ward M, Kaufmann M, Turton J, Fagan E, James D, et al. Community and hospital spread of Escherichia coli producing CTX-M extendedspectrum $\beta$-lactamases in the UK. J Antimicrob Chemother. 2004;54(4):735-43.

50. Hackman HK, Brown CA, Twum-Danso K, Bu K. Antibiotic Resistance Profile of CTX-M-type Extended-Spectrum Beta-Lactamases in Escherichia coli and Klebsiella pneumoniae in Accra, Ghana. J Nat Sci Res. 2014;4(12):24-29.

51. Sana T, Rami K, Racha B, Fouad D, Marcel A, Hassan M, et al. Detection of genes TEM, OXA, SHV and CTX-M in 73 clinical isolates of Escherichia coli producers of extended spectrum Betalactamases and determination of their susceptibility to antibiotics. Int Arab J Antimicrob Agents. 2011;1(1). https:// doi.org/10.3823/704

52. Upadhyay S, Hussain A, Mishra S, Maurya AP, Bhattacharjee A, Joshi SR. Genetic environment of plasmid mediated CTX-M-15 extended Spectrum Beta-lactamases from clinical and food borne Bacteria in north-eastern India PLoS One. 2015;10(9):e0138056.

53. Shin J, Ko KS. Comparative study of genotype and virulence in CTX-Mproducing and non-extended-spectrum- $\beta$-lactamase-producing Klebsiella pneumoniae isolates. Antimicrob Agents Chemother. 2014:58(4):2463-7.

54. Ensor V, Shahid M, Evans J, Hawkey P. Occurrence, prevalence and genetic environment of CTX-M $\beta$-lactamases in Enterobacteriaceae from Indian hospitals. J Antimicrob Chemother. 2006;58(6):1260-3.

55. Tan TY, Ng SY. Comparison of Etest, Vitek and agar dilution for susceptibility testing of colistin. Clin Microbiol Infect. 2007 May;13(5):541-4.

56. European Committee on Antimicrobial Susceptibility Testing. Breakpoint tables for interpretation of MICs and zone diameters. Version 6.0: EUCAST; 2016. http://www.eucast.org/fileadmin/src/media/PDFs/EUCAST_files/ Breakpoint tables/v 6.0_Breakpoint table.pdf. Accessed 20 Feb 2018

57. European Committee on Antimicrobial Susceptibility Testing. Recommendations for MIC determination of colistin (polymyxin E) as recommended by the joint CLSI-EUCAST Polymyxin Breakpoints Working Group EUCAST. http://www.eucast.org/fileadmin/src/media/PDFs/EUCAST_ files/General_documents/Recommendations_for_MIC_determination_of_ colistin_March_2016.pdf. Accessed 20 Feb 2018.

58. Desta K, Woldeamanuel Y, Azazh A, Mohammod H, Desalegn D, Shimelis D, et al. High gastrointestinal colonization rate with extended-Spectrum $\beta$ lactamase-producing Enterobacteriaceae in hospitalized patients: emergence of Carbapenemase-Producing K. pneumoniae in Ethiopia. PLoS One. 2016;11(8):e0161685. 
59. Christiaens G, Ciccarella Y, Damas P, Hayette MP, Melin P, Nys M, De Mol P. Prospective survey of digestive tract colonization with enterobacteriaceae that produce ESBLs in intensive care units. J Hosp Infect. 2006;62(3):386-8.

60. Falagas M, Karageorgopoulos DE. Extended-spectrum $\beta$-lactamaseproducing organisms. J Hosp Infect. 2009;73(4):345-54.

61. Pritsch M, Zeynudin A, Messerer M, Baumer S, Liegl G, Schubert S, Löscher T, Hoelscher M, Belachew T, Rachow A, Wieser A. First repot on blaNDM-1producing Acinetobacter baumannii in three clinical isolates from Ethiopia. BMC Infect Dis. 2017;17(1):180.

62. Hawkey PM, Jones AM. The changing epidemiology of resistance. J Antimicrob Chemother. 2009 Sep;64(Suppl 1):i3-10.

Ready to submit your research? Choose BMC and benefit from:

- fast, convenient online submission

- thorough peer review by experienced researchers in your field

- rapid publication on acceptance

- support for research data, including large and complex data types

- gold Open Access which fosters wider collaboration and increased citations

- maximum visibility for your research: over $100 \mathrm{M}$ website views per year

At $\mathrm{BMC}$, research is always in progress.

Learn more biomedcentral.com/submissions 